\title{
Phenolic Profile, Antioxidant Activity, and Enzyme Inhibitory Properties of Limonium delicatulum (Girard) Kuntze and Limonium quesadense Erben
}

\author{
A. Ruiz-Riaguas, ${ }^{1}$ G. Zengin, ${ }^{2}$ K.I. Sinan, ${ }^{2}$ C. Salazar-Mendías, ${ }^{3}$ \\ and E.J. Llorent-Martínez ${ }^{1}$ \\ ${ }^{1}$ Department of Physical and Analytical Chemistry, Faculty of Experimental Sciences, University of Jaén, \\ Campus Las Lagunillas, E-23071 Jaén, Spain \\ ${ }^{2}$ Department of Biology, Science Faculty, Selcuk University, Campus, Konya, Turkey \\ ${ }^{3}$ Department of Animal Biology, Plant Biology and Ecology, Faculty of Experimental Sciences, University of Jaén, \\ Campus Las Lagunillas, E-23071 Jaén, Spain
}

Correspondence should be addressed to E.J. Llorent-Martínez; ellorent@ujaen.es

Received 29 August 2019; Revised 9 January 2020; Accepted 25 January 2020; Published 11 March 2020

Academic Editor: Maria P. Robalo

Copyright $\odot 2020$ A. Ruiz-Riaguas et al. This is an open access article distributed under the Creative Commons Attribution License, which permits unrestricted use, distribution, and reproduction in any medium, provided the original work is properly cited.

\begin{abstract}
In this work, we report the phytochemical composition and bioactive potential of methanolic and aqueous extracts of leaves from Limonium delicatulum (Girard) Kuntze and Limonium quesadense Erben. The characterization and quantitation of individual phytochemicals were performed with liquid chromatography with diode array and electrospray-tandem mass spectrometry detection. Myricetin glycosides were abundant in L. delicatulum, whereas L. quesadense was rich in gallo(epi)catechin-O-gallate. Total phenolics, flavonols, and flavonoids were assayed with conventional methods. Antioxidant and radical scavenging assays (phosphomolybdenum, DPPH, ABTS, CUPRAC, FRAP, and metal chelating activity), as well as enzyme inhibitory assays (acetylcholinesterase, butyrylcholinesterase, tyrosinase, amylase, glucosidase, and lipase), were performed to evaluate the potential bioactivity. The methanolic extracts of both species presented higher phenolic content and bioactivity than the aqueous extracts. Overall, $L$. quesadense extracts exhibited the most potent activity for most assays, representing a potential source of bioactive compounds for the pharmaceutical and food industries.
\end{abstract}

\section{Introduction}

Plants represent a rich source of many bioactive compounds, particularly polyphenols, which are well known for their high antioxidant activity and various health benefits. As a result, an increasing number of plant species are constantly used in folk medicine. In fact, several recent studies have focused on the enzyme inhibitory properties of plant extracts as an interesting approach to prevent different chronic diseases, such as inflammation, diabetes mellitus, Alzheimer, and cancer. Here, we present an investigation concerning two species of the genus Limonium: L. delicatulum (Girard) Kuntze and L. quesadense Erben.

Limonium Miller is a genus that belongs to the Plumbaginaceae family, specifically to the Staticoideae subfamily.
There are two subgenera (Pteroclados and Limonium) with different sections-depending on the authors-and at least 10 subsections $[1,2]$. There are between 350 and 470 species, mainly distributed in the western Mediterranean region [2-4]. This genus comprises perennial species and, rarely, annual herbaceous plants. Limonium species usually grow in arid or semiarid areas, occupying small isolated spaces over gypsic or saline soils. There are numerous local endemic species and, due to their isolation, many of them are threatened and protected species. Some species are cultivated as ornamental plants, whereas others have important medicinal properties $[5,6]$. Research on species of this genus has revealed important bioactivity concerning the free radical scavenging $[7,8]$, antioxidant $[5,9-11]$, anti-inflammatory [10], antibacterial [12], antimicrobial [9], and 
antiviral [13] properties. The main phenolics identified as responsible for these activities were gallic acid, epigallocatechin gallate, and myricetin and isorhamnetin flavonoids $[7,8,10,11,14]$.

L. delicatulum is an Iberian-North African endemism [3], growing up to $100 \mathrm{~cm}$. Leaves are green, usually ovate to elliptic or obovate $(3.5-15 \mathrm{~cm}$ length $\times 2-5 \mathrm{~cm}$ width), with 4-10 lateral nerves. It blooms from February to October depending on the altitude, developing shoots of $20-90 \mathrm{~cm}$ with spikes of 5-25 mm and spikelets of 4-5 mm. It inhabits coastal and inland saline habitats between 0 and 800 m.a.s.l. It is not considered a threatened species [15] and its chemical composition and bioactivity have been scarcely studied. Its antioxidant activity has been reported [16, 17], as well as total phenolics, flavonoids, tannins, and antimicrobial activity [17]. However, the detailed phytochemical composition and potential enzyme inhibitory activities have not been reported so far.

L. quesadense is endemic to the province of Jaén (southeastern Iberian Peninsula, Spain) of 35-60 cm. Leaves are green-bluish to green-violetish, oblanceolate to spathulate $(4-12 \mathrm{~cm}$ length $\times 1.5-3 \mathrm{~cm}$ width), with 4 (rarely 6) lateral nerves. It blooms from June to August, developing shoots of $20-50 \mathrm{~cm}$ with spikes of 7-20 $\mathrm{mm}$ and spikelets of $4.5-5 \mathrm{~mm}$. It takes part in continental halophytic vegetation and gypsophyte scrubs in the Guadiana Menor valley between 500 and 700 m.a.s.l. It is regarded as a threatened plant under the category of "endangered" (EN) $[15,18]$. To date, there are no studies concerning the phytochemical composition and bioactivity of this species.

Taking into account the lack of information concerning the two target species-as well as the reports of the bioactivity of other Limonium species-this research aims at providing information concerning the phenolic composition of leaves of $L$. delicatulum and L. quesadense, examining their antioxidant activity (radical scavenging, reducing power, and metal chelating) and enzyme inhibitory properties (against acetylcholinesterase, butyrylcholinesterase, tyrosinase, amylase, glucosidase, and lipase).

\section{Materials and Methods}

2.1. Sample Preparation. Leaves of $L$. quesadense and $L$. delicatulum were collected at the Native Flora Garden of the University of Jaén (Jaén, Andalusia, Spain; $37^{\circ} 47^{\prime} 18.879^{\prime \prime} \mathrm{N}$ $3^{\circ} 46^{\prime} 31.583^{\prime \prime} \mathrm{W}, 427 \mathrm{~m}$ a.s.l.) in September 2018. Samples are stored at the Herbarium of the University of Jaén. Photographs of both species are shown in Figure 1 .

The taxonomical classification was confirmed by botanist Dr. Carlos Salazar-Mendías. Samples were washed with Milli-Q water and extracted by two different procedures:

(i) Ultrasound-assisted extraction with $\mathrm{MeOH}$ : leaves were lyophilized (ModulyoD-23, Thermo Savant; Waltham, MA USA) and powdered; $2.5 \mathrm{~g}$ of sample was extracted with $50 \mathrm{~mL}$ of $\mathrm{MeOH}$ in an ultrasonic liquid processor (Qsonica Sonicator; Newtown, CT, USA; power of $55 \mathrm{~W}$ and frequency of $20 \mathrm{kHz}$ ) at $50 \%$ power for $10 \mathrm{~min}$. (ii) Decoction: $2.5 \mathrm{~g}$ of sample (fresh and powdered) was extracted with $150 \mathrm{~mL}$ of boiling Milli-Q water for 30 minutes.

Both extracts were filtered through Whatman No. 1 filters, and the solvent was evaporated under reduced pressure in a Hei-Vap Precision Rotary Evaporator (Heidolph; Schwabach; Germany) at $40^{\circ} \mathrm{C}$. Dried extracts (DE) were stored at $-20^{\circ} \mathrm{C}$ until analysis.

2.2. HPLC Analysis. Dried extracts (5-10 mg) were redissolved in $1 \mathrm{~mL}$ of $\mathrm{MeOH}$ and filtered with $0.45 \mu \mathrm{m}$ nylon filters, and $10 \mu \mathrm{L}$ of the sample was injected. The HPLC system was an Agilent Series 1100 with a G1315B diode array detector. The separation of the compounds was performed with a reversed-phase Luna Omega Polar $\mathrm{C}_{18}$ column $(150 \times 3.0 \mathrm{~mm}$ and $5 \mu \mathrm{m}$ particle size; Phenomenex $)$ with a Polar $\mathrm{C}_{18}$ Security Guard cartridge (Phenomenex) of $4 \times 3.0 \mathrm{~mm}$. The HPLC system was connected to an ion trap mass spectrometer (Esquire 6000, Bruker Daltonics) with an electrospray interface. Chromatographic conditions have been previously detailed [19]. All standards required to perform phenolic quantitation were purchased from SigmaAldrich (Madrid, Spain); individual stock solutions were prepared in methanol $(\mathrm{MeOH}$, LC-MS grade, >99.9\%; Sigma). LC-MS grade acetonitrile $\left(\mathrm{CH}_{3} \mathrm{CN}, 99 \%\right.$; LabScan; Dublin, Ireland) and ultrapure water (Milli-Q Water $\mathrm{Pu}$ rification System; Millipore; Milford, MA, USA) were also used.

2.3. Total Phenolic Content (TPC) and Total Flavonoid Content (TFC). TPC and TFC were determined using the Folin-Ciocalteu and $\mathrm{AlCl}_{3}$ assays, respectively [20]. Results were expressed as gallic acid equivalents (mg GAEs/g extract) and rutin equivalents (mg REs/g extract) for the respective assays.

2.4. Determination of Antioxidant and Enzyme Inhibitory Effects. The metal chelating, phosphomolybdenum, FRAP, CUPRAC, ABTS, and DPPH activities of the extracts were assessed following the methods described by Uysal et al. [20]. The antioxidant activities were reported as trolox equivalents, whereas EDTA was used for metal chelating assay. The possible inhibitory effects of the extracts against cholinesterases (AChE (E.C. 3.1.1.7) from Electrophorus electricus (electric eel) and BChE (E.C. 3.1.1.8) from equine serum, by Ellman's method), tyrosinase (from mushroom, E.C. 1.14.18.1), $\alpha$-amylase (from porcine pancreas, E.C. 3.2.1.1), $\alpha$-glucosidase (E.C. 3.2.1.20), and lipase (from porcine pancreas, E.C 3.1.1.3) were evaluated using standard in vitro bioassays [21].

2.5. Data Analysis. Bioactive compounds and biological activity data were prepared for univariate and multivariate statistical analysis. Firstly, one-way ANOVA followed by post hoc test, namely, Tukey's multiple range was performed under Xlstat 2018 to investigate significant differences 


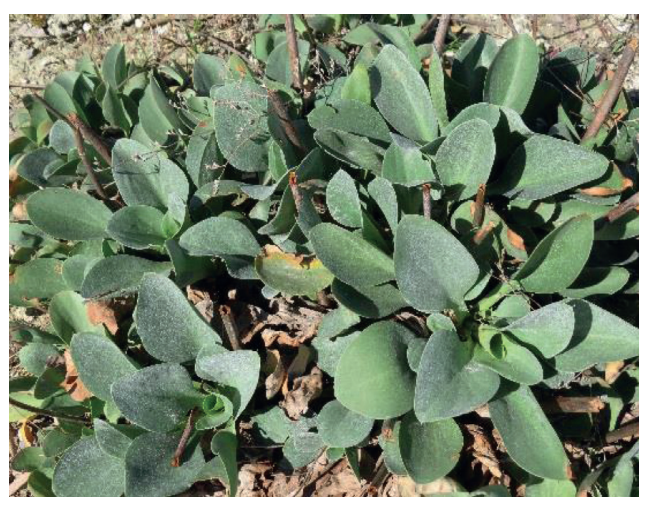

(a)

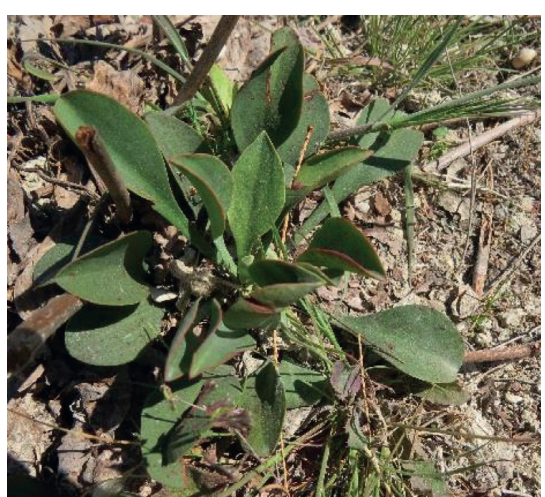

(b)

Figure 1: Photographs of (a) L. delicatulum and (b) L. quesadense.

$(p<0.05)$ between the studied samples. The data were subjected to unsupervised multivariate analysis PCA and HCA using R software v. 3.5.1 for the discrimination between the extracts and their classification according to biological activities. Finally, the relationship between biological activities and phenolic classes based on the estimation of Pearson's correlation coefficients were conducted.

\section{Results and Discussion}

3.1. Phytochemical Characterization. The characterization of phytochemicals was performed by HPLC-ESI-MS ${ }^{\mathrm{n}}$ using negative and positive ion modes. Base peak chromatograms are shown in Figure 2, whereas the characterization of compounds is detailed in Table 1 .

Compound $\mathbf{1}$ was identified as the $\mathrm{HCl}$ adduct of a disaccharide (dihexoside) due to its fragmentation pattern [22]. Compound 2, with deprotonated molecular ion at $\mathrm{m} / \mathrm{z}$ 191 and base peak at $m / z 111$, was identified as citric acid.

Compound 4, with $[\mathrm{M}-\mathrm{H}]^{-}$at $m / z 169$ and base peak at $\mathrm{m} / z 125$, was identified as gallic acid by comparison with an analytical standard. Compound 3 , after the loss of $80 \mathrm{Da}$ (sulfate moiety), displayed fragment ions at $\mathrm{m} / z$ 331, 169, and 125, typical of galloyl hexoside. Compound 7 also presented gallic acid in its fragmentation pattern and was tentatively characterized as a derivative. Compound 9 was tentatively characterized as prodelphinidin dimer B-type gallate ( 2 units of gallo(epi)catechin) based on bibliographic information [23].

Compounds 14, 16, and $\mathbf{1 8}$ presented $[\mathrm{M}-\mathrm{H}]^{-}$at $\mathrm{m} / z 457$ and fragment ions at $m / z 331,305,169$, and 125 , consistent with gallo(epi)catechin-O-gallate isomers [23]. Compound $\mathbf{1 5}$ was characterized as a dimer.

Compound 8 exhibited fragment ions at $\mathrm{m} / \mathrm{z} 153$ and $109 / 108$, which corresponded to protocatechuic acid (comparison with an analytical standard), so it was characterized as a derivative.

Compound 11, identified using positive ion mode, suffered the neutral loss of $162 \mathrm{Da}$, yielding cyanidin at $\mathrm{m} / \mathrm{z}$ 287 , so it was characterized as cyanidin 3-glucoside [24].

Several myricetin derivatives were characterized in the analyzed extracts. In all of them, myricetin was observed at $\mathrm{m} / z 317$ (main fragment ions at $\mathrm{m} / z 179$ and 151). The following neutral losses were observed in compounds 17, 19, 20, 23, 35, and 36: $152 \mathrm{Da}$ (galloyl moiety), $146 \mathrm{Da}$ (deoxyhexoside), $162 \mathrm{Da}$ (hexoside), $308 \mathrm{Da}$ (rutinoside). We could not elucidate the exact structure of compounds $\mathbf{2 6}$ and 30, so they were characterized as myricetin derivatives.

Following the same neutral losses than myricetin, several quercetin $(21,22,24,31,38$, and 39) and kaempferol $(28,29$, and 37) derivatives were characterized. Quercetin and kaempferol aglycones were detected at $\mathrm{m} / \mathrm{z} 301$ and 285, respectively.

Compound 27 suffered the neutral loss of $80 \mathrm{Da}$ (sulfate) to yield the lignan syringaresinol at $\mathrm{m} / z$ 417, which was identified by the fragment ions at $m / z 181,166$, and 151 [25]. Compound 34 was also characterized as a sulfate adduct of a lignan, pinoresinol [25].

Compound 32 was characterized as delphinidin due to the $303 \longrightarrow 257$ fragmentation using positive ion mode. With an additional hexoside moiety, $\mathbf{2 5}$ was characterized as delphinidin-O-hexoside [24].

Finally, compound 33, with deprotonated molecular ion at $m / z 477$, suffered the neutral loss of $146 \mathrm{Da}$ (deoxyhexoside) to yield mearnsetin at $m / z 331$ (main fragment ion at $\mathrm{m} / \mathrm{z} 316$ ) [26].

3.2. Quantitation of Phenolic Compounds. We quantified 16 compounds in the methanolic and aqueous extracts of $L$. quesadense and $L$. delicatulum. The results are summarised in Table 2. Total individual phenolic content (TIPC) was defined as the sum of all the individual compounds that were quantified by HPLC-DAD (phenolic acids at $320 \mathrm{~nm}$ and flavonoids at $350 \mathrm{~nm}$ ).

L. quesadense presented higher TIPC ( 54 and $32 \mathrm{mg} / \mathrm{g}$ $\mathrm{DE}$ for $\mathrm{MeOH}$ and $\mathrm{H}_{2} \mathrm{O}$ extracts, respectively) than $L$. delicatulum (17 and $3.1 \mathrm{mg} / \mathrm{g} \mathrm{DE}$ for $\mathrm{MeOH}$ and $\mathrm{H}_{2} \mathrm{O}$ extracts, respectively). In both cases, methanol extracts presented the highest concentration of phenolics due to the highest solubility of flavonoids in $\mathrm{MeOH}$ compared to water. The most abundant compounds in L. delicatulum $\mathrm{MeOH}$ extract were myricetin glycosides (compounds 19, 20, and 23), which have been previously reported as 


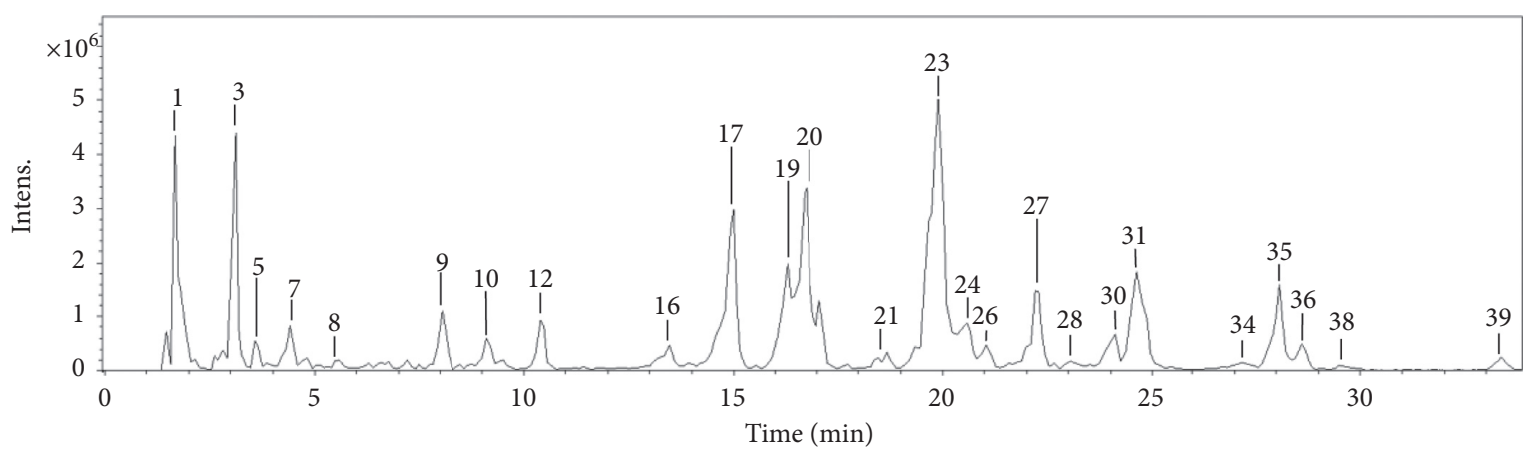

(a)

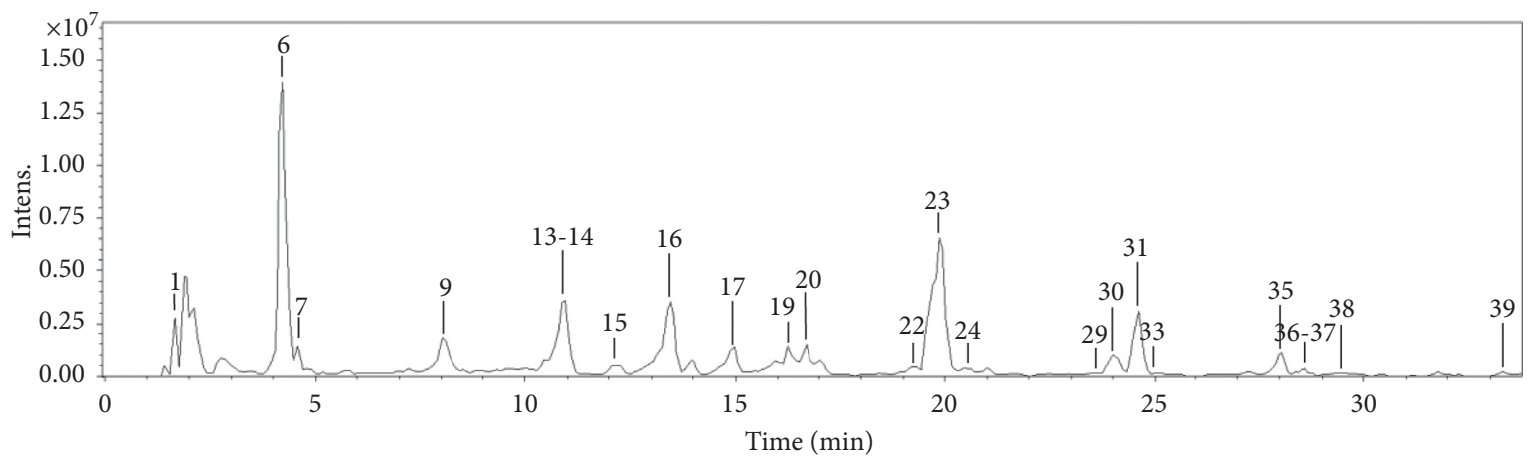

(b)

FIGURE 2: HPLC-ESI-MS ${ }^{\mathrm{n}}$ base peak chromatograms of the methanolic extracts of (a) L. delicatulum and (b) L. quesadense.

bioactive compounds in L. densiflorum [10]. On the other hand, the most abundant compounds in L. quesadense extracts were 15 and 16 (gallo(epi)catechin-O-gallate monomer and dimer), followed by myricetin glycosides. Epigallocatechin gallate has been previously reported as the potential mainly responsible for the bioactive properties of L. brasiliense and L. algarvense $[11,14]$.

3.3. Antioxidant Properties. The majority of plant-based aromatic natural products are phenols, which comprise flavonoids, tannins, flavonols, flavanols, and anthocyanins, among others. In the present study, the different prepared extracts have been screened for the presence of phenolics, flavonols, and flavonoids. Indeed, all extracts showed a good level of phenolics, followed by flavonols and flavonoids (Table 3), observing higher levels in L. quesadense than L. delicatulum. In addition, $\mathrm{MeOH}$ extracts presented higher levels of phenolics than aqueous extracts. These results are in agreement with TIPC quantified by chromatography. The methanolic extract of $L$. quesadense possessed the highest TPC (172 $\pm 4 \mathrm{mg}$ GAE/ $\mathrm{g} \cdot \mathrm{DE})$ and flavonol (74 $\pm 3 \mathrm{mg} \mathrm{RE} / \mathrm{g} \cdot \mathrm{DE})$. However, flavonoids were most abundant in the $\mathrm{MeOH}$ extract of $L$. delicatulum $(42.1 \pm 0.8 \mathrm{mg} \mathrm{RE} / \mathrm{g} \cdot \mathrm{DE})$.

Moreover, a series of antioxidant assays were conducted on the extracts of both Limonium species, namely, total antioxidant capacity (phosphomolybdenum), radical scavenging (ABTS and DPPH), reducing power (CUPRAC and FRAP), and metal chelating. These results are presented in Tables 3 and 4 .
In terms of total antioxidant capacity, the most potent extract was the methanolic extract of $L$. quesadense $(5.1 \pm 0.4 \mathrm{mmol} \mathrm{TE} / \mathrm{g} \cdot \mathrm{DE})$. However, it is essential to point out that there is no statistical difference between this extract and the aqueous and methanolic extracts of $L$. delicatulum. Interestingly, the methanolic extract of $L$. quesadense displayed the highest activity in reducing power and radical scavenging assays. It showed significant activity with ABTS $(510 \pm 30 \mathrm{mg} \mathrm{TE} / \mathrm{g} \cdot \mathrm{DE})$ and DPPH $(620 \pm 10 \mathrm{mg} \mathrm{TE} / \mathrm{g} \cdot \mathrm{DE})$, CUPRAC ( $940 \pm 10 \mathrm{mg}$ TE/g.DE), and FRAP (520 $\pm 10 \mathrm{mg} \mathrm{TE} /$ g.DE). The most abundant flavonoid identified in the methanolic extract of $L$. quesadense was gallo(epi)catechin-O-gallate $\left(26 \pm 1 \mathrm{mg} \cdot \mathrm{g}^{-1} \cdot \mathrm{DE}\right)$. Thus, it can be extrapolated that this compound, along with its dimer, might be mainly responsible for the observed antioxidant properties.

In contrast to the aforementioned antioxidant assays, the results obtained in the metal chelating assay classified the aqueous extract of $L$. delicatulum as the most effective metal chelator, with a significant activity of $28.43 \pm 0.01 \mathrm{mg}$ EDTAE/g.DE. Considering the quantitation analysis of phenolic compounds of all extracts (Table 2), it can be said that there exists a good correlation between the antioxidant results and the quantitation of polyphenols. As ample evidence, the total quantified phenolic content (TIPC) with HPLC reported the methanolic extract of L. quesadense as most rich in flavonoids $\left(54 \pm 1 \mathrm{mg} \cdot \mathrm{g}^{-1} \mathrm{DE}\right)$ which as discussed above presented the highest antioxidant property. In this sense, the observed significant antioxidant properties could be attributed mainly to the presence of compounds containing galloyl moieties, such a gallo(epi)catechin-O- 
TABLE 1: Characterization of phenolics in L. delicatulum and L. quesadense extracts.

\begin{tabular}{|c|c|c|c|c|c|c|}
\hline No. & $\begin{array}{c}\mathrm{t}_{R} \\
(\mathrm{~min})\end{array}$ & {$[\mathrm{M}-\mathrm{H}]^{-} m / z$} & $m / z$ (\% base peak) & $\begin{array}{c}\text { Assigned } \\
\text { identification }\end{array}$ & L. delicatulum & L. quesadense \\
\hline 1 & 1.7 & 377 & $\begin{array}{c}\mathrm{MS}^{2} \text { [377]: } 341 \text { (100) } \\
\mathrm{MS}^{3}[377 \longrightarrow 341]: 179(100), 131(14), 113 \text { (18) } \\
\mathrm{MS}^{4}[377 \longrightarrow 341 \longrightarrow 179]: 161(42), 143(93), 119(100)\end{array}$ & Disaccharide ( $\mathrm{HCl}$ adduct) & $\checkmark$ & $\checkmark$ \\
\hline 2 & 2.0 & 191 & $\operatorname{MS}^{2}[191]: 173(25), 127(19), 111(100)$ & Citric acid & & $\mathrm{J}^{\mathrm{b}}$ \\
\hline 3 & 3.1 & 411 & $\operatorname{MS}^{2}$ [411]: 331 (7), $241(100), 169(14), 125(6)$ & Galloyl hexoside (sulfate adduct) & $\checkmark$ & \\
\hline 4 & 3.3 & 169 & $\mathrm{MS}^{2}$ [169]: $125(100)$ & Gallic acid & $\sqrt{ }^{\mathrm{b}}$ & $J^{\mathrm{b}}$ \\
\hline 5 & 3.6 & 439 & $\begin{array}{l}\mathrm{MS}^{2} \text { [439]: } 241(100) \\
\mathrm{MS}^{3}[439 \longrightarrow 241]: 223(96), 139(100), 165(16)\end{array}$ & Unknown & $\checkmark$ & \\
\hline 6 & 4.3 & 379 & $\operatorname{MS}^{2}$ [379]: $379(100), 241(20)$ & Unknown & & $\checkmark$ \\
\hline 7 & 4.5 & 325 & $\begin{array}{l}\operatorname{MS}^{2}[325]: 169(100), 125(9) \\
\operatorname{MS}^{3}[325 \longrightarrow 169]: 125(100)\end{array}$ & Gallic acid derivative & $\checkmark$ & $\checkmark$ \\
\hline 8 & 5.6 & 365 & $\begin{array}{c}\operatorname{MS}^{2}[365]: 321(68), 153(100) \\
\operatorname{MS}^{3}[365 \longrightarrow 153]: 109(100), 108(61)\end{array}$ & Protocatechuic acid derivative & $\checkmark$ & \\
\hline 9 & 8.0 & 761 & $\begin{array}{l}\operatorname{MS}^{2}[761]: 609(89), 593(96), 575(74), 423(100) \\
\operatorname{MS}^{3}[761 \longrightarrow 423]: 297(50), 283(100), 243(28)\end{array}$ & $\begin{array}{l}\text { Prodelphinidin dimer B-type gallate } \\
\qquad(2 \text { units (epi)GC) }\end{array}$ & $\sqrt{ }^{\mathrm{a}}$ & $\checkmark$ \\
\hline 10 & 9.1 & 443 & $\operatorname{MS}^{2}[443]: 275(24), 245(100), 167(27)$ & Unknown & $\checkmark$ & \\
\hline 11 & 9.5 & $449(+)$ & $\mathrm{MS}^{2}$ [449]:431 (8), 288(18), 287(100) & Cyanidin 3-glucoside & $\sqrt{ }^{\mathrm{a}}$ & \\
\hline 12 & 10.4 & 759 & $\begin{array}{c}\operatorname{MS}^{2}[759]: 481(100), 423(96), 301(87) \\
\text { MS }^{3}[759 \longrightarrow 423]: 297(84), 283(50), 243(100)\end{array}$ & Unknown & $\checkmark$ & \\
\hline 13 & 11.0 & 363 & $\operatorname{MS}^{2}[363]: 363(100), 241(10)$ & Unknown & & $\checkmark$ \\
\hline 14 & 11.0 & 457 & $\begin{array}{c}\mathrm{MS}^{2}[457]: 331(20), 305(17), 193(85), 169(100) \\
\operatorname{MS}^{3}[457 \longrightarrow 169]: 125(100) \\
\operatorname{MS}^{2}[913]: 761(100), 423(60)\end{array}$ & Gallo(epi)catechin-O-gallate isomer & & $\checkmark^{\mathrm{a}}$ \\
\hline 15 & 12.3 & 913 & $\begin{array}{c}\operatorname{MS}^{3}[913 \longrightarrow 761]: 423(100), 609(90), 305 \text { (49) } \\
\operatorname{MS}^{4}[913 \longrightarrow 761 \longrightarrow 423]: 297(100), 253(49), 405(33)\end{array}$ & Gallo(epi)catechin-O-gallate dimer & & $\checkmark$ \\
\hline 16 & 13.3 & 457 & $\begin{array}{c}\mathrm{MS}^{2}[457]: 331(22), 305(13), 169(100) \\
\operatorname{MS}^{3}[457 \longrightarrow 169]: 125(100) \\
\operatorname{MS}^{2}[631]: 479(80), 317(100)\end{array}$ & Gallo(epi)catechin-O-gallate isomer & $\checkmark$ & $\checkmark$ \\
\hline 17 & 15.0 & 631 & $\begin{array}{c}\operatorname{MS}^{3}[631 \longrightarrow 479]: 317(100), 316(91), 179(9) \\
\text { MS }^{4}[631 \longrightarrow 479 \longrightarrow 317]: 271(100), 179(54), 151 \text { (18) }\end{array}$ & Myricetin-galloyl-hexoside & $\checkmark$ & $\checkmark$ \\
\hline 18 & 15.4 & 457 & $\begin{array}{c}\operatorname{MS}^{2}[457]: 331(16), 305(15), 169(100) \\
\operatorname{MS}^{3}[457 \longrightarrow 169]: 125(100)\end{array}$ & Gallo(epi)catechin-O-gallate isomer & & $\sqrt{ }^{\mathrm{b}}$ \\
\hline 19 & 16.2 & 625 & $\begin{array}{l}\mathrm{MS}^{2} \text { [625]: } 317(100), 316(89) \\
\left.\mathrm{MS}^{3} \text { [625 } \longrightarrow 317\right]: 271(100), 179(48), 151 \text { (26) }\end{array}$ & Myricetin-O-rutinoside & $\checkmark$ & $\checkmark$ \\
\hline 20 & 16.6 & 479 & $\begin{array}{c}\mathrm{MS}^{2}[479]: 317(79), 316(100), 179(5) \\
\operatorname{MS}^{3}[479 \longrightarrow 317]: 271(100), 179(67), 151(16) \\
\operatorname{MS}^{2} \text { [615]: } 463(100), 301(33)\end{array}$ & Myricetin- $O$-hexoside & $\checkmark$ & $\checkmark$ \\
\hline 21 & 18.5 & 615 & $\begin{array}{c}\mathrm{MS}^{3}[615 \longrightarrow 463]: 301(100) \\
\mathrm{MS}^{4}[615 \longrightarrow 463 \longrightarrow 301]: 179(75), 151(100)\end{array}$ & Quercetin-galloyl-hexoside & $\checkmark$ & $\sqrt{ }^{\mathrm{b}}$ \\
\hline 22 & 19.2 & 609 & $\begin{array}{c}\mathrm{MS}^{2} \text { [609]: } 301(100) \\
\operatorname{MS}^{3}[609 \longrightarrow 301]: 271(98), 179(76), 151(100)\end{array}$ & Rutin & & $\checkmark$ \\
\hline 23 & 19.9 & 463 & $\begin{array}{c}\mathrm{MS}^{2}[463]: 317(77), 316(100), 179(10) \\
\operatorname{MS}^{3}[463 \longrightarrow 316]: 271(100), 179(60), 151 \text { (18) }\end{array}$ & Myricetin-O-deoxyhexoside & $\checkmark$ & $\checkmark$ \\
\hline 24 & 20.6 & 463 & $\begin{array}{c}\mathrm{MS}^{2}[463]: 301(100), 151(5) \\
\mathrm{MS}^{3}[463 \longrightarrow 301]: 255(21), 179(70), 151 \text { (100) }\end{array}$ & Quercetin-O-hexoside & $\checkmark$ & $\checkmark$ \\
\hline 25 & 20.6 & $465(+)$ & $\begin{array}{c}\mathrm{MS}^{2}[465]: 303(100) \\
\mathrm{MS}^{3}[465 \longrightarrow 303]: 257(100), 137 \text { (39) }\end{array}$ & Delphinidin-O-hexoside & & $\checkmark$ \\
\hline 26 & 21.0 & 659 & $\begin{array}{c}\mathrm{MS}^{2}[659]: 317(85), 316(100) \\
\operatorname{MS}^{3}[659 \longrightarrow 316]: 271(100), 179(74), 151(47) \\
\operatorname{MS}^{2} \text { [497]: } 417(100)\end{array}$ & Myricetin derivative & $\sqrt{ }^{\mathrm{a}}$ & \\
\hline 27 & 22.2 & 497 & $\begin{array}{c}\operatorname{MS}^{3}[497 \longrightarrow 417]: 371(14), 181(100), 166(50), 151 \text { (24) } \\
\operatorname{MS}^{4}[497 \longrightarrow 417 \longrightarrow 181]: 166(100)\end{array}$ & Syringaresinol (sulfate adduct) & $\checkmark$ & \\
\hline 28 & 23.0 & 593 & $\begin{array}{c}\mathrm{MS}^{2} \text { [593]: } 286(20), 285(100) \\
\operatorname{MS}^{3}[595 \longrightarrow 285]: 257(100), 239(76), 213(75), 151 \text { (45) }\end{array}$ & Kaempferol-O-rutinoside & $\checkmark$ & \\
\hline 29 & 23.5 & 599 & $\begin{array}{c}\mathrm{MS}^{2}[599]: 313(100), 285(94) \\
\mathrm{MS}^{3}[599 \longrightarrow 313]: 169(100) \\
\quad \operatorname{MS}^{2}[549]: 505(100)\end{array}$ & Kaempferol-O-galloyl-hexoside & & $\checkmark$ \\
\hline 30 & 24.1 & 549 & $\begin{array}{c}\mathrm{MS}^{3}[549 \longrightarrow 505]: 317(44), 316(100) \\
\mathrm{MS}^{4}[549 \longrightarrow 505 \longrightarrow 316]: 271(100), 179(57), 151 \text { (16) }\end{array}$ & Myricetin derivative & $\mathfrak{J}^{\mathrm{a}}$ & $\checkmark$ \\
\hline 31 & 24.6 & 447 & $\begin{array}{c}\operatorname{MS}^{2}[447]: 301(100), 179(5) \\
\operatorname{MS}^{3}[447 \longrightarrow 301]: 179(91), 151(100)\end{array}$ & Quercetin-O-deoxyhexoside & $\checkmark$ & $\checkmark$ \\
\hline 32 & 24.7 & $303(+)$ & $\operatorname{MS}^{2}$ [303]: $257(100)$ & Delphinidin & $\sqrt{ }^{\mathrm{a}}$ & \\
\hline 33 & 24.9 & 477 & $\begin{array}{c}\mathrm{MS}^{2} \text { [477]: } 331(100) \\
\operatorname{MS}^{3}[477 \longrightarrow 331]: 316(100), 315(57)\end{array}$ & Mearnsetin- $O$-deoxyhexoside & & $\sqrt{ }^{\mathrm{a}}$ \\
\hline 34 & 27.2 & 437 & $\begin{array}{c}\mathrm{MS}^{2} \text { [437]: } 357(100), 151(39) \\
\mathrm{MS}^{3}[437 \longrightarrow 357]: 342(48), 151 \text { (100), } 136(40)\end{array}$ & Pinoresinol (sulfate adduct) & $\checkmark$ & \\
\hline 35 & 28.0 & 615 & $\begin{array}{c}\mathrm{MS}^{2} \text { [615]: } 317(100) \\
\mathrm{MS}^{3}[615 \longrightarrow 317]: 179(100), 151(40)\end{array}$ & Myricetin-O-galloyl-deoxyhexoside & $\sqrt{ }^{\mathrm{a}}$ & $\checkmark$ \\
\hline
\end{tabular}


TABle 1: Continued.

\begin{tabular}{|c|c|c|c|c|c|c|}
\hline No. & $\begin{array}{c}\mathrm{t}_{R} \\
(\mathrm{~min}) \\
\end{array}$ & {$[\mathrm{M}-\mathrm{H}]^{-} m / z$} & $m / z$ (\% base peak) & $\begin{array}{c}\text { Assigned } \\
\text { identification }\end{array}$ & L. delicatulum & L. quesadense \\
\hline 36 & 28.6 & 615 & $\begin{array}{c}\mathrm{MS}^{2}[615]: 317(100) \\
\mathrm{MS}^{3}[615 \longrightarrow 317]: 179(100), 151(54)\end{array}$ & Myricetin-O-galloyl-deoxyhexoside & $\mathfrak{V}^{\mathrm{a}}$ & $\checkmark$ \\
\hline 37 & 28.6 & 431 & $\begin{array}{c}\operatorname{MS}^{2}[431]: 286(17), 285(100), 284(25), 255(10) \\
\operatorname{MS}^{3}[431 \longrightarrow 285]: 257(82), 255(100), 197(39) \\
\operatorname{MS}^{2}[533]: 489(100)\end{array}$ & Kaempferol-O-deoxyhexoside & & $\checkmark$ \\
\hline 38 & 29.5 & 533 & $\begin{array}{c}\mathrm{MS}^{3}[533 \longrightarrow 489]: 447(19), 301(100) \\
\mathrm{MS}^{4}[533 \longrightarrow 489 \longrightarrow 301]: 271(100), 179(22), 151(42) \\
\operatorname{MS}^{2}[599]: 301(100)\end{array}$ & Quercetin derivative & $\checkmark^{\mathrm{a}}$ & $\checkmark$ \\
\hline 39 & 33.4 & 599 & $\begin{array}{l}\mathrm{MS}^{3}[599 \longrightarrow 301]: 179(81), 151(100) \\
\mathrm{MS}^{4}[599 \longrightarrow 301 \longrightarrow 179]: 151(100)\end{array}$ & Quercetin-O-galloyl-deoxyhexoside & $\checkmark$ & $\checkmark$ \\
\hline
\end{tabular}

${ }^{\mathrm{a} O n l y}$ in $\mathrm{MeOH}$ extract; ${ }^{\mathrm{b}}$ only in $\mathrm{H}_{2} \mathrm{O}$ extract.

TABle 2: Contents ( $\left.\mathrm{mg} \mathrm{g}^{-1} \mathrm{DE}\right)$ of the main phenolic compounds present in L. delicatulum and L. quesadense extracts.

\begin{tabular}{|c|c|c|c|c|c|}
\hline \multirow{2}{*}{ No. } & \multirow{2}{*}{ Assigned identification } & \multicolumn{2}{|c|}{ L. delicatulum } & \multicolumn{2}{|c|}{ L. quesadense } \\
\hline & & $\mathrm{MeOH}$ & $\mathrm{H}_{2} \mathrm{O}$ & $\mathrm{MeOH}$ & $\mathrm{H}_{2} \mathrm{O}$ \\
\hline \multicolumn{6}{|c|}{ Phenolic acids } \\
\hline 3 & Galloyl hexoside & $0.67 \pm 0.01$ & $1.91 \pm 0.1$ & - & - \\
\hline 7 & Gallic acid derivative & $0.84 \pm 0.02$ & - & - & - \\
\hline Total & & $1.51 \pm 0.02$ & $1.9 \pm 0.1$ & & \\
\hline \multicolumn{6}{|c|}{ Flavonoids } \\
\hline 9 & Prodelphinidin dimer & $0.58 \pm 0.01^{\mathrm{c}}$ & - & $5.10 \pm 0.01^{\mathrm{b}}$ & $6.3 \pm 0.3^{\mathrm{a}}$ \\
\hline 15 & Gallo(epi)catechin-O-gallate dimer & - & - & $10.0 \pm 0.7$ & - \\
\hline 16 & Gallo(epi)catechin-O-gallate & $1.7 \pm 0.2^{\mathrm{c}}$ & - & $26 \pm 1^{\mathrm{a}}$ & $15.0 \pm 0.6^{\mathrm{b}}$ \\
\hline 17 & Myricetin-galloyl-hexoside & $1.2 \pm 0.1^{\mathrm{a}}$ & - & $0.89 \pm 0.07^{\mathrm{b}}$ & $0.69 \pm 0.05^{\mathrm{c}}$ \\
\hline 18 & Gallo(epi)catechin-O-gallate & - & - & - & $2.68 \pm 0.05$ \\
\hline $19+20$ & Myricetin glycosides & $4.40 \pm 0.01^{\mathrm{a}}$ & $0.30 \pm 0.02^{\mathrm{d}}$ & $2.22 \pm 0.06^{\mathrm{b}}$ & $1.41 \pm 0.08^{\mathrm{c}}$ \\
\hline 23 & Myricetin-O-deoxyhexoside & $5.1 \pm 0.1^{\mathrm{b}}$ & $0.64 \pm 0.05^{\mathrm{d}}$ & $7.5 \pm 0.4^{\mathrm{a}}$ & $4.1 \pm 0.1^{\mathrm{c}}$ \\
\hline 24 & Quercetin-O-hexoside & $0.54 \pm 0.05^{\mathrm{a}}$ & $0.21 \pm 0.02^{\mathrm{b}}$ & - & $0.21 \pm 0.02^{\mathrm{b}}$ \\
\hline 30 & Myricetin derivative & - & - & $0.50 \pm 0.07$ & $0.39 \pm 0.02$ \\
\hline 31 & Quercetin-O-deoxyhexoside & $0.93 \pm 0.04^{\mathrm{b}}$ & - & $1.37 \pm 0.06^{\mathrm{a}}$ & $0.75 \pm 0.04^{\mathrm{c}}$ \\
\hline 35 & Myricetin-O-galloyl-deoxyhexoside & $0.56 \pm 0.03^{\mathrm{a}}$ & - & $0.48 \pm 0.01^{\mathrm{b}}$ & $0.24 \pm 0.01^{\mathrm{c}}$ \\
\hline 36 & Myricetin-O-galloyl-deoxyhexoside & $0.28 \pm 0.01$ & - & - & $0.25 \pm 0.01$ \\
\hline 39 & Quercetin-O-galloyl-deoxyhexoside & $0.18 \pm 0.01$ & - & - & - \\
\hline Total & & $15.5 \pm 0.3^{c}$ & $1.15 \pm 0.05^{\mathrm{d}}$ & $54 \pm 1^{a}$ & $32.0 \pm 0.7^{b}$ \\
\hline TIPC & & $17.0 \pm 0.3^{\mathrm{c}}$ & $3.1 \pm 0.1^{\mathrm{d}}$ & $54 \pm 1^{a}$ & $32.0 \pm 0.7^{b}$ \\
\hline
\end{tabular}

Values are means \pm SD of three parallel measurements. Means in the same line not sharing the same letter are significantly different $(p<0.05)$.

TABLE 3: Total bioactive components, total antioxidant capacity (by phosphomolybdenum assay), and radical scavenging abilities of $L$. delicatulum and L. quesadense extracts.

\begin{tabular}{|c|c|c|c|c|c|c|c|}
\hline Plant species & Solvents & $\begin{array}{c}\text { Total phenolic } \\
\text { content } \\
\text { (mg GAE/g.DE) }\end{array}$ & $\begin{array}{c}\text { Total flavonol } \\
\text { content } \\
\text { (mg RE/g.DE) }\end{array}$ & $\begin{array}{c}\text { Total flavonoid } \\
\text { content } \\
\text { (mg RE/g.DE) }\end{array}$ & $\begin{array}{l}\text { Phosphomolybdenum } \\
\text { (mmol TE/g.DE) }\end{array}$ & $\begin{array}{c}\text { ABTS } \\
(\mathrm{mg} \mathrm{TE} / \mathrm{g} \mathrm{DE})\end{array}$ & $\begin{array}{c}\text { DPPH } \\
(\mathrm{mg} \mathrm{TE} / \mathrm{g} \mathrm{DE})\end{array}$ \\
\hline L. delicatulum & $\begin{array}{c}\mathrm{MeOH} \\
\mathrm{H}_{2} \mathrm{O}\end{array}$ & $\begin{array}{c}151 \pm 1^{\mathrm{b}} \\
31.1 \pm 0.4^{\mathrm{c}}\end{array}$ & $\begin{array}{c}55 \pm 2^{\mathrm{b}} \\
1.08 \pm 0.03^{\mathrm{d}}\end{array}$ & $\begin{array}{c}42.1 \pm 0.8^{\mathrm{a}} \\
5.80 \pm 0.09^{\mathrm{d}}\end{array}$ & $\begin{array}{c}4.5 \pm 0.6^{\mathrm{a}} \\
0.67 \pm 0.04^{\mathrm{b}}\end{array}$ & $\begin{array}{c}360 \pm 10^{\mathrm{b}} \\
53 \pm 8^{\mathrm{d}}\end{array}$ & $\begin{array}{l}470 \pm 10^{\mathrm{b}} \\
56 \pm 1^{\mathrm{d}}\end{array}$ \\
\hline L. quesadense & $\begin{array}{c}\mathrm{MeOH} \\
\mathrm{H}_{2} \mathrm{O}\end{array}$ & $\begin{array}{l}172 \pm 4^{\mathrm{a}} \\
152 \pm 1^{\mathrm{b}}\end{array}$ & $\begin{array}{c}74 \pm 3^{\mathrm{a}} \\
10.4 \pm 0.2^{\mathrm{c}}\end{array}$ & $\begin{array}{c}30.8 \pm 0.5^{\mathrm{b}} \\
12.98 \pm 0.07^{\mathrm{c}}\end{array}$ & $\begin{array}{l}5.1 \pm 0.4^{\mathrm{a}} \\
4.6 \pm 0.7^{\mathrm{a}}\end{array}$ & $\begin{array}{l}510 \pm 30^{\mathrm{a}} \\
248 \pm 6^{\mathrm{c}}\end{array}$ & $\begin{array}{l}620 \pm 10^{\mathrm{a}} \\
428 \pm 8^{\mathrm{c}}\end{array}$ \\
\hline
\end{tabular}

Values expressed are means \pm SD of three parallel measurements. GAE: gallic acid equivalent; RE: rutin equivalent; TE: trolox equivalent. Means in the same column not sharing the same letter are significantly different $(p<0.05)$.

TABLE 4: Reducing power and metal chelating abilities of L. delicatulum and L. quesadense extracts.

\begin{tabular}{lcccc}
\hline Plant species & Solvents & CUPRAC $(\mathrm{mg}$ TE/g.DE) & FRAP (mg TE/g.DE) & Metal chelating activity (mg EDTAE/g.DE) \\
\hline \multirow{2}{*}{ L. delicatulum } & $\mathrm{MeOH}$ & $853 \pm 5^{\mathrm{b}}$ & $470 \pm 10^{\mathrm{b}}$ & $26.74 \pm 0.01^{\mathrm{b}}$ \\
& $\mathrm{H}_{2} \mathrm{O}$ & $94 \pm 2^{\mathrm{d}}$ & $62.5 \pm 0.6^{\mathrm{d}}$ & $28.43 \pm 0.01^{\mathrm{a}}$ \\
\hline \multirow{2}{*}{ L. quesadense } & $\mathrm{MeOH}$ & $940 \pm 10^{\mathrm{a}}$ & $520 \pm 10^{\mathrm{a}}$ & $19.43 \pm 0.01^{\mathrm{d}}$ \\
& $\mathrm{H}_{2} \mathrm{O}$ & $640 \pm 10^{\mathrm{c}}$ & $431 \pm 5^{\mathrm{c}}$ & $22.30 \pm 0.01^{\mathrm{c}}$ \\
\hline
\end{tabular}

Values expressed are means \pm SD of three parallel measurements. TE: trolox equivalent; EDTAE: EDTA equivalent. Means in the same column not sharing the same letter are significantly different $(p<0.05)$. 
TABLe 5: Enzyme inhibitory properties of L. delicatulum and L. quesadense extracts.

\begin{tabular}{|c|c|c|c|c|c|c|c|}
\hline Plant species & Solvents & $\begin{array}{c}\text { AChE inhibition } \\
\text { (mg GALAE/ } \\
\text { g.DE) }\end{array}$ & $\begin{array}{c}\text { BChE inhibition } \\
\text { (mg GALAE/ } \\
\text { g.DE) }\end{array}$ & $\begin{array}{l}\text { Tyrosinase } \\
\text { (mg KAE/ } \\
\text { g.DE) }\end{array}$ & $\begin{array}{c}\text { Amylase } \\
\text { (mmol ACAE/ } \\
\text { g.DE) }\end{array}$ & $\begin{array}{c}\text { Glucosidase } \\
\text { (mmol ACAE/ } \\
\text { g.DE) }\end{array}$ & $\begin{array}{c}\text { Lipase } \\
(\mathrm{mg} \mathrm{OE} / \\
\mathrm{g} \cdot \mathrm{DE})\end{array}$ \\
\hline \multirow{2}{*}{ L. delicatulum } & $\mathrm{MeOH}$ & $4.8 \pm 0.7^{\mathrm{a}}$ & $3.5 \pm 0.4^{\mathrm{a}}$ & $155.87 \pm 0.01^{\mathrm{a}}$ & $0.95 \pm 0.03^{\mathrm{b}}$ & $2.70 \pm 0.01^{\mathrm{c}}$ & $27 \pm 4^{\mathrm{b}}$ \\
\hline & $\mathrm{H}_{2} \mathrm{O}$ & $1.0 \pm 0.2^{\mathrm{c}}$ & na & $18.87 \pm 0.01^{\mathrm{d}}$ & $0.08 \pm 0.00^{\mathrm{c}}$ & $2.74 \pm 0.01^{\mathrm{a}}$ & na \\
\hline \multirow{2}{*}{ L. quesadense } & $\mathrm{MeOH}$ & $4.3 \pm 0.2^{\mathrm{a}}$ & $2.63 \pm 0.02^{b}$ & $155.27 \pm 0.01^{b}$ & $1.00 \pm 0.02^{\mathrm{b}}$ & $2.72 \pm 0.01^{\mathrm{b}}$ & $65 \pm 7^{a}$ \\
\hline & $\mathrm{H}_{2} \mathrm{O}$ & $1.7 \pm 0.2^{\mathrm{b}}$ & $0.86 \pm 0.01^{\mathrm{c}}$ & $135.34 \pm 0.01^{c}$ & $1.5 \pm 0.3^{\mathrm{a}}$ & na & na \\
\hline
\end{tabular}

Values expressed are means \pm SD of three parallel measurements. GALAE: galantamine equivalent; KAE: kojic acid equivalent; ACAE: acarbose equivalent; OE: orlistat equivalent; na: not active. Means in the same column not sharing the same letter are significantly different $(p<0.05)$.

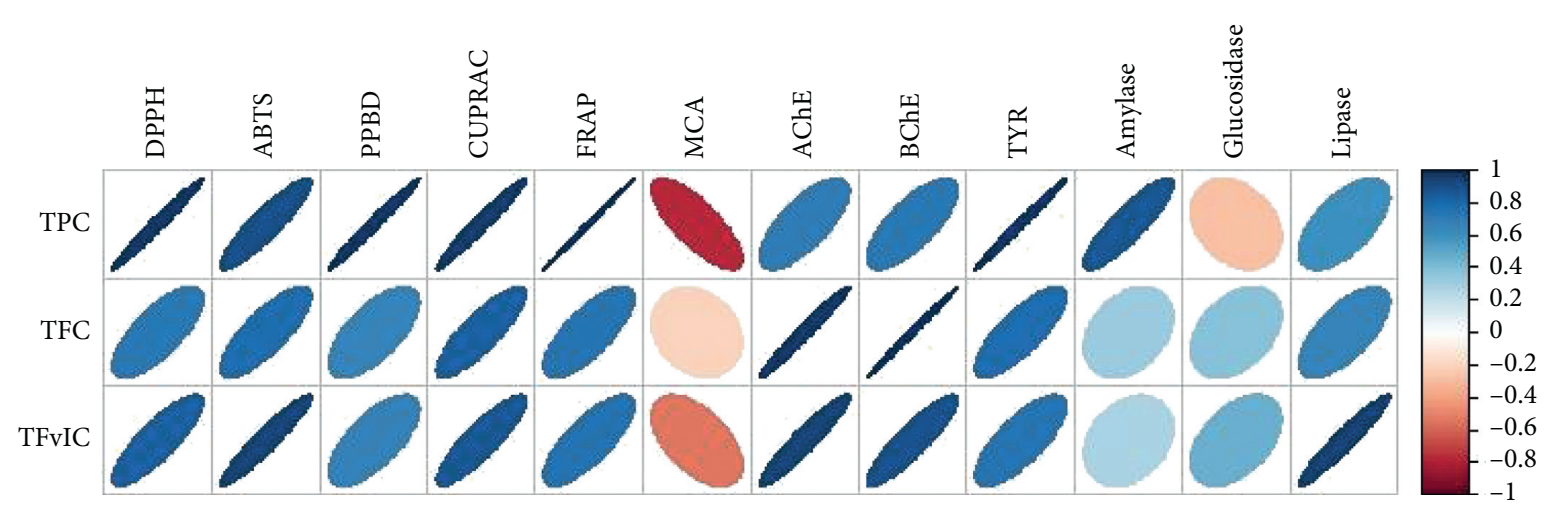

(a)

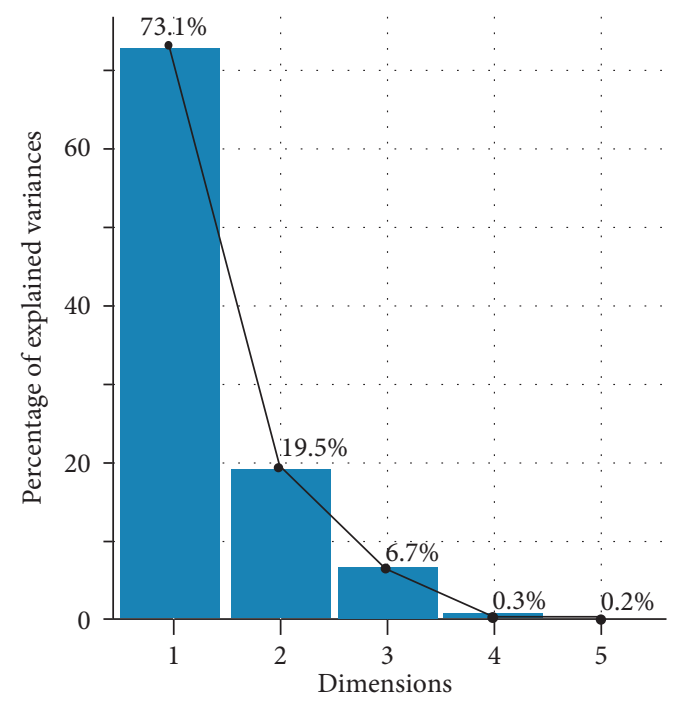

(b)

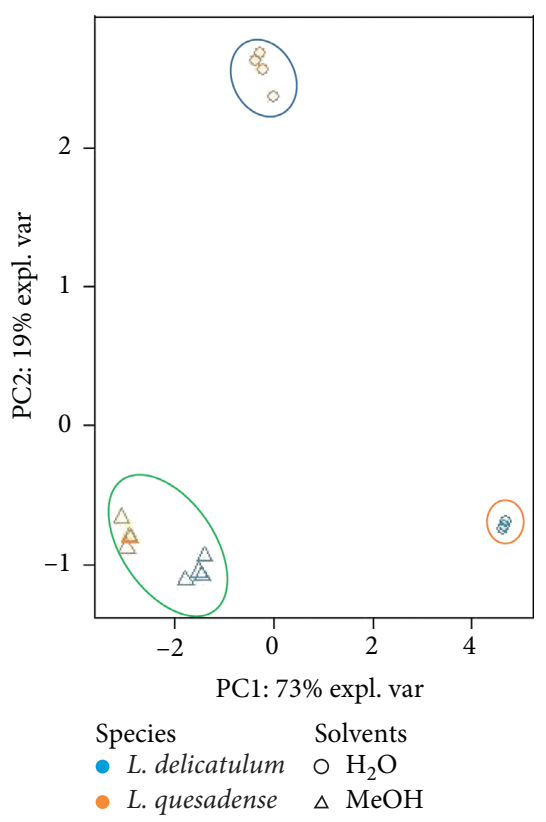

(c)

Figure 3: Continued. 


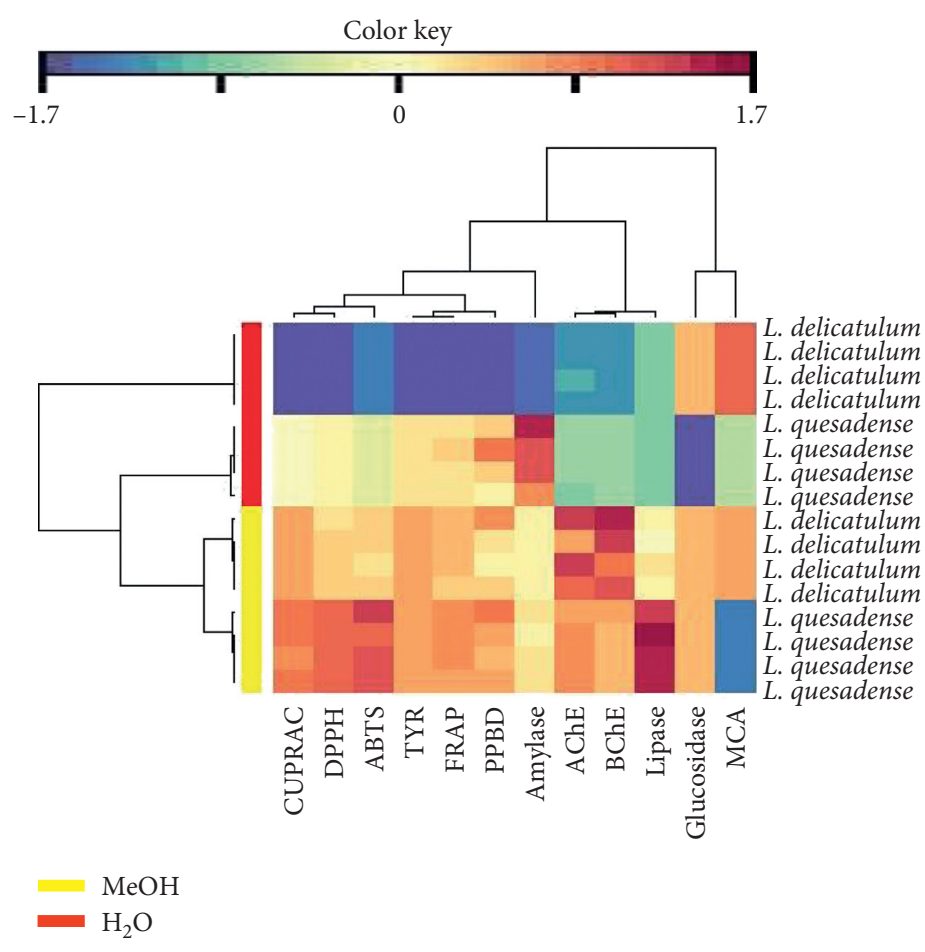

(d)

FIGURE 3: Relationship between total bioactive compounds and biological activities and multivariate analysis of Limonium species. (a) Pearson's correlation heatmap; (b) screen plot of explained variance by PCA components; (c) score plot of principal components 1 and 2; (d) hierarchical cluster dendrogram of extracts. The color box indicates the standardized biological activities of each extract. The red, yellow, and blue colors indicate high, middle, and low bioactivity, respectively.

gallate dimer. Our findings were also supported by several researchers $[27,28]$.

3.4. Enzyme Inhibitory Effects. Enzymes are the main targets to control the constantly emerging global health issues [29]. As an example, tyrosinase is an important enzyme involved in the melanogenesis process during which the pigment, melanin, is produced [30]. However, the inhibitor of tyrosinase, kojic acid, which is used inexhaustibly by the pharmaceutical and cosmetic industries, represents various side effects [31]. Furthermore, the drug orlistat, which is the only clinically approved pharmacologic agent against pancreatic lipase, is associated with considerable side effects [32]. Thus, there is a dire need to search for new and safer enzymatic inhibitors for future pharmaceutical development. Accordingly, this present study is in line with the current trend and has screened the prepared extracts from the two Limonium species against $\alpha$-amylase, glucosidase, acetylcholinesterase (AChE), butyrylcholinesterase (BChE), tyrosinase, and lipase.

Tyrosinase inhibitors from the methanolic extract of L. delicatulum $(155.87 \pm 0.01 \mathrm{mg} \mathrm{KAE} / \mathrm{g} \cdot \mathrm{DE})$ and lipase inhibitors from the methanolic extract of $L$. quesadense $(65 \pm 7 \mathrm{mg}$ OE/g.DE) seemed promising candidates. The methanolic and aqueous extracts of L. delicatulum and L. quesadense were screened for their inhibitory activities on both AChE and BChE (Table 5).
We observed that the methanolic extract of L. delicatulum exhibited the highest activity $(4.8 \pm 0.7 \mathrm{mg}$ GALAE/g.DE); nevertheless, there is no statistical difference between the latter extract and the methanolic extract of L. quesadense. Hence, the two mentioned extracts represent the most potent cholinesterase inhibitors. Furthermore, the aqueous extract of $L$. quesadense was the most active inhibitor for $\alpha$-amylase $(1.5 \pm 0.3 \mathrm{mmol} \mathrm{ACAE} / \mathrm{g} \cdot \mathrm{DE})$. On the other hand, in terms of glucosidase enzymatic assay, we observed the aqueous extract of $L$. delicatulum to be more potent $(2.74 \pm 0.01 \mathrm{mmol}$ ACAE/ g.DE) followed by the methanolic extract of $L$. quesadense $(2.72 \pm 0.01 \mathrm{mmol} \mathrm{ACAE} / \mathrm{g} \cdot \mathrm{DE})$ and the methanolic extract of L. delicatulum $(2.70 \pm 0.01 \mathrm{mmol}$ ACAE/g.DE). Further data collected in this present study showed that the methanolic extract of $L$. quesadense exhibited the most effective lipase inhibitor. A substantial amount of reports showed that several plant metabolites are prospective pancreatic lipase inhibitors. Principally, it is projected that the presence of galloyl moiety of flavan-3-ols is essential for lipase inhibition [33]. Indeed, the methanolic extract of $L$. quesadense contained the highest levels of gallo(epi)catechin-O-gallate and its dimer, as well as myricetin-O-hexoside (Table 2) which might be linked to the significant lipase activity. It is noteworthy to point out that although the methanolic extract of L. quesadense possessed the highest bioactive components, we did not observe the most significant activity in all enzymatic assays. These results display that there may not always a correlation between polyphenol contents and enzymatic inhibition assays. 
3.5. Unsupervised Multivariate Data Analysis of Biological Activities of Limonium Extracts. The analysis of Limonium species extracts encompassing 12 biological activities justified the employment of multivariate data analysis tools. Thus, with the help of unsupervised PCA and hierarchical clustering analysis, the biological activity data allowed for discrimination between the different extracts.

The first two principal components showed $73.1 \%$ and $19.5 \%$ of the total variance, respectively, suggesting only the two components could outline $90 \%$ information of the original data. As presented in Figure 3, the extracts were clearly classified into three clusters. Likewise, hierarchical cluster analysis (HCA) based on the concept of Euclidean similarity measure and Wards as linkage rule between the extracts confirmed the PCA results.

The $\mathrm{MeOH}$ extracts of two studied species were close enough. That suggested both extracts have similar properties against all evaluated biological activities. Otherwise, as opposed to $\mathrm{MeOH}$ extracts, the $\mathrm{H}_{2} \mathrm{O}$ extracts were different. It allowed to better discriminate the two species. Accordingly, $L$. delicatulum and $L$. quesadense had different properties against all biological activities excepted AChE, $\mathrm{BChE}$, and lipase; nevertheless, a more significant difference between the two species was obtained with MCA and amylase assays. Therefore, it can be concluded that L. delicatulum was most active against MCA while $L$. quesadense showed better amylase inhibitory activity.

\section{Conclusions}

The phenolic composition and bioactive properties of leaves of $L$. delicatulum and $L$. quesadense have been examined. $L$. delicatulum was rich in myricetin glycosides, whereas some of the most abundant compounds in L. quesadense were gallo(epi)catechin-O-gallate and its dimer. The presence of these compounds has been previously reported in other Limonium species and has been suggested as the main responsible for the bioactivity of Limonium extracts. In general, methanolic extracts presented the highest amounts of phenolics, along with the highest bioactive properties, although the most potent activities were observed in L. quesadense leaves. Not only the antioxidant activity was evaluated, but also the enzyme inhibitory properties against several key enzymes. The overall results indicate that leaves of $L$. quesadense may represent an interesting source of bioactive compounds. As L. quesadense is a threatened plant that is not currently protected by law, its cultivation on gypsic soils could be tested under the permission of the authorities by using seeds collected in the wild. It may also be an economic impulse for the population of semiarid areas in Jaén province.

\section{Data Availability}

The data used to support the findings of this study are available from the corresponding author upon request.

\section{Disclosure}

The abstract of this manuscript has been presented in the conference SEQA 2019.

\section{Conflicts of Interest}

The authors declare that there are no conflicts of interest regarding the publication of this paper.

\section{Acknowledgments}

Technical and human support provided by CICT of Universidad de Jaén (UJA, MINECO, Junta de Andalucía, FEDER) is gratefully acknowledged. Junta de Andalucía and Universidad de Jaén are acknowledged for the possibility of farming and collecting the plant species.

\section{References}

[1] M. D. Lledo, M. B. Crespo, M. F. Fay, and M. W. Chase, "Molecular phylogenetics of Limonium and related genera (Plumbaginaceae): biogeographical and systematic implications," American Journal of Botany, vol. 92, no. 7, pp. 11891198, 2005.

[2] K. Kubitzki, J. G. Rohwer, and V. Bittrich, The Families and Genera of Vascular Plants. Flowering Plants-Dicotyledons, vol. 2, Springer, Berlin, Germany, 1993.

[3] G. Domina, The Information Resource for Euro-Mediterranean Plant Diversity, Euro+Med Plantbase, Berlin, Germany, 2011, http://ww2.bgbm.org/EuroPlusMed.

[4] M. Erben, "Limonium Mill," in Flora Iberica, S. Castroviejo, C. Aedo, S. Cirujano et al., Eds., vol. 3, pp. 2-143, Real Jardín Botánico-CSIC, Madrid, Spain, 1993.

[5] D. Geng, X. Chi, Q. Dong, and F. Hu, “Antioxidants screening in Limonium aureum by optimized on-line HPLC-DPPH assay," Industrial Crops and Products, vol. 67, pp. 492-497, 2015.

[6] L.-C. Lin and C.-J. Chou, "Flavonoids and phenolics from Limonium sinense," Planta Medica, vol. 66, no. 4, pp. 382-383, 2000.

[7] A. P. Murray, S. Rodriguez, M. A. Frontera, M. A. Tomas, and M. C. Mulet, "Antioxidant metabolites from Limonium brasiliense (boiss.) Kuntze," Zeitschrift für Naturforschung C, vol. 59, no. 7-8, pp. 477-480, 2004.

[8] Y. Aniya, C. Miyagi, A. Nakandakari, S. Kamiya, N. Imaizumi, and T. Ichiba, "Free radical scavenging action of the medicinal herb Limonium wrightii from the Okinawa islands," Phytomedicine, vol. 9, no. 3, pp. 239-244, 2002.

[9] F. Faten Medini, R. Ksouri, H. Falleh, W. Megdiche, N. Trabelsi, and C. Abdelly, "Effects of physiological stage and solvent on polyphenol composition, antioxidant and antimicrobial activities of Limonium densiflorum," Journal of Medicinal Plants Research, vol. 5, no. 31, pp. 6719-6730, 2011.

[10] F. Medini, S. Bourgou, K. Lalancette et al., "Phytochemical analysis, antioxidant, anti-inflammatory, and anticancer activities of the halophyte Limonium densiflorum extracts on human cell lines and murine macrophages," South African Journal of Botany, vol. 99, pp. 158-164, 2015.

[11] M. J. Rodrigues, A. Soszynski, A. Martins et al., "Unravelling the antioxidant potential and the phenolic composition of different anatomical organs of the marine halophyte Limonium algarvense," Industrial Crops and Products, vol. 77, pp. 315-322, 2015.

[12] A. Blainski, B. Gionco, A. G. Oliveira et al., "Antibacterial activity of Limonium brasiliense (Baicuru) against multidrugresistant bacteria using a statistical mixture design," Journal of Ethnopharmacology, vol. 198, pp. 313-323, 2017. 
[13] F. Medini, J. Legault, A. Pichette, C. Abdelly, and R. Ksouri, "Antiviral efficacy of Limonium densiflorum against HSV-1 and influenza viruses," South African Journal of Botany, vol. 92, pp. 65-72, 2014.

[14] A. de Oliveira Caleare, A. Hensel, J. C. P. Mello et al., "Flavan3-ols and proanthocyanidins from Limonium brasiliense inhibit the adhesion of Porphyromonas gingivalis to epithelial host cells by interaction with gingipains," Fitoterapia, vol. 118, pp. 87-93, 2017.

[15] C. Salazar and M. L. Lendínez, "Limonium Mill," in Claves de La Flora Vascular de Andalucía Oriental, G. Blanca, B. Cabezudo, M. Cueto et al., Eds., Universidades de Granada, Granada, Spain, 2011.

[16] A. Souid, L. Bellani, C. Magné et al., "Physiological and antioxidant responses of the sabkha biotope halophyte Limonium delicatulum to seasonal changes in environmental conditions," Plant Physiology and Biochemistry, vol. 123, pp. 180-191, 2018.

[17] F. Medini, H. Fellah, R. Ksouri, and C. Abdelly, "Total phenolic, flavonoid and tannin contents and antioxidant and antimicrobial activities of organic extracts of shoots of the plant Limonium delicatulum," Journal of Taibah University for Science, vol. 8, no. 3, pp. 216-224, 2014.

[18] F. Guerrero, G. Parra, F. Jiménez-Gómez et al., "Ecological studies in Alto Guadalquivir wetlands: a first step towards the application of conservation plans," Limnetica, vol. 25, no. 1, pp. 95-106, 2006.

[19] E. J. Llorent-Martínez, G. Zengin, D. Lobine, L. MolinaGarcía, A. Mollica, and M. F. Mahomoodally, "Phytochemical characterization, in vitro and in silico approaches for three Hypericum species," New Journal of Chemistry, vol. 42, no. 7, pp. 5204-5214, 2018.

[20] S. Uysal, G. Zengin, M. Locatelli et al., "Cytotoxic and enzyme inhibitory potential of two Potentilla species (P. speciosa L. and $P$. reptans Willd.) and their chemical composition," Frontiers in Pharmacology, vol. 8, p. 290, 2017.

[21] D. M. Grochowski, S. Uysal, A. Aktumsek et al., "In vitro enzyme inhibitory properties, antioxidant activities, and phytochemical profile of Potentilla thuringiaca," Phytochemistry Letters, vol. 20, pp. 365-372, 2017.

[22] G. Verardo, I. Duse, and A. Callea, "Analysis of underivatized oligosaccharides by liquid chromatography/electrospray ionization tandem mass spectrometry with post-column addition of formic acid," Rapid Communications in Mass Spectrometry, vol. 23, no. 11, pp. 1607-1618, 2009.

[23] L. Bresciani, L. Calani, M. Cossu et al., "(Poly)phenolic characterization of three food supplements containing 36 different fruits, vegetables and berries," PharmaNutrition, vol. 3, no. 2, pp. 11-19, 2015.

[24] V. Spínola, E. J. Llorent-Martínez, S. Gouveia, and P. C. Castilho, "Myrica faya: a new source of antioxidant phytochemicals," Journal of Agricultural and Food Chemistry, vol. 62, no. 40, pp. 9722-9735, 2014.

[25] P. C. Eklund, M. J. Backman, L. Å. Kronberg, A. I. Smeds, and R. E. Sjöholm, "Identification of lignans by liquid chromatography-electrospray ionization ion-trap mass spectrometry," Journal of Mass Spectrometry, vol. 43, no. 1, pp. 97-107, 2008.

[26] J. Han, M. Ye, X. Qiao, M. Xu, B.-R. Wang, and D.-A. Guo, "Characterization of phenolic compounds in the Chinese herbal drug Artemisia annua by liquid chromatography coupled to electrospray ionization mass spectrometry," Journal of Pharmaceutical and Biomedical Analysis, vol. 47, no. 3, pp. 516-525, 2008.
[27] B. Badhani, N. Sharma, and R. Kakkar, "Gallic acid: a versatile antioxidant with promising therapeutic and industrial applications," RSC Advances, vol. 5, no. 35, pp. 27540-27557, 2015.

[28] M. Muzolf-Panek, A. Gliszczyńska-Świgło, H. Szymusiak, and B. Tyrakowska, "The influence of stereochemistry on the antioxidant properties of catechin epimers," European Food Research and Technology, vol. 235, no. 6, pp. 1001-1009, 2012.

[29] M. Vujanović, G. Zengin, S. Đurović, P. Mašković, A. Cvetanović, and M. Radojković, "Biological activity of extracts of traditional wild medicinal plants from the Balkan Peninsula," South African Journal of Botany, vol. 120, pp. 213-218, 2019.

[30] T. Pillaiyar, M. Manickam, and V. Namasivayam, "Skin whitening agents: medicinal chemistry perspective of tyrosinase inhibitors," Journal of Enzyme Inhibition and Medicinal Chemistry, vol. 32, no. 1, pp. 403-425, 2017.

[31] G. Zengin, S. Uysal, R. Ceylan, A. Aktumsek, C. M. N. Picot, and M. F. Mahomoodally, "Phenolic constituent, antioxidative and tyrosinase inhibitory activity of Ornithogalum narbonense L. from Turkey: a phytochemical study," Industrial Crops and Products, vol. 70, pp. 1-6, 2015.

[32] T. Buchholz and M. Melzig, "Polyphenolic compounds as pancreatic lipase inhibitors," Planta Medica, vol. 81, no. 10, pp. 771-783, 2015.

[33] A. T. M. A. Rahim, Y. Takahashi, and K. Yamaki, "Mode of pancreatic lipase inhibition activity in vitro by some flavonoids and non-flavonoid polyphenols," Food Research International, vol. 75, pp. 289-294, 2015. 